\title{
Olfactory mucosal microstructural changes in a rat model of acute rhinosinusitis with dysosmia
}

\author{
J. Ye ${ }^{1}$, J.P. He ${ }^{2}$ and Z.J. Liu ${ }^{1}$ \\ 'Department of Otorhinolaryngology, \\ The 1st Affiliated Hospital of Nanchang University, Nanchang, China \\ ${ }^{2}$ Department of Otorhinolaryngology, \\ Quanzhou 180 Hospital of People Liberation Army, Quanzhou, China \\ Corresponding author: J. Ye \\ E-mail: jingyec@126.com
}

Genet. Mol. Res. 13 (2): 3859-3868 (2014)

Received August 9, 2013

Accepted December 11, 2013

Published May 16, 2014

DOI http://dx.doi.org/10.4238/2014.May.16.11

\begin{abstract}
This study aimed to examine olfactory mucosal changes in a rat model of acute rhinosinusitis and to determine how acute rhinosinusitis induces dysosmia. Total 100 rats were divided into the experimental $(\mathrm{N}=80)$ and control $(\mathrm{N}=20)$ groups. An acute rhinosinusitis model was established in the experimental rats. From the experimental group, 20 rats each were sacrificed at 1,2,3, or 4 weeks. The control group was sacrificed at 1 week without inoculation. Olfaction was tested by the buried food pellet test before killing the rats, and the duration until the pellet was recorded and analyzed by a statistical software program. Mature olfactory receptor neurons (ORNs) and olfactory ensheathing cells (OECs) were examined. The durations spent by the experimental group in the BFPT were $402.9 \pm 9.3,453.7 \pm 7.3,351.9$ \pm 8.9 , and $278.7 \pm 8.1 \mathrm{~s}$ at $1,2,3$, and 4 weeks, respectively. These durations were greater than that of the control group $(178.3 \pm 6.6 \mathrm{~s})$, and the difference was statistically significant $(\mathrm{P}<0.01)$. A reduction in ORNs and thinning of the epithelium were detected in the experimental group, most notably in the 2nd week and less in the fourth week. OECs were reduced in the 1st week,
\end{abstract}


increased in the 2nd week, and almost completely recovered in the fourth week. In addition, some growing OECs were detected in the experimental group. Acute rhinosinusitis decreased both the mature ORNs and OECs, but OECs increased faster than the ORNs.

Key words: Acute rhinosinusitis; Dysosmia; Olfactory receptor neurons; Olfactory ensheathing cells

\section{INTRODUCTION}

Olfactory receptor neurons (ORNs) are continuously generated throughout life, and dysosmia is often caused by damage to the olfactory pathway, especially the ORNs (Shipley et al., 2008). Olfactory ensheathing cells (OECs) are a special type of glial cells that are known as the only cells that are capable of sustainable regeneration of ORNs (Raisman and Li, 2007). The removal of the olfactory bulb and olfactory nerve transection can cause ORN apoptosis (Shetty et al., 2005). In addition, OECs can improve the microenvironment of neurons, induce remyelination of axons to guide fresh axons through the glial scar and transitional zone of the central-peripheral nervous system, and promote synapse formation, all of which improve olfactory function. In this study, we conducted experiments based on a rat acute rhinosinusitis model to detect changes in mature ORNs and OECs in the olfactory mucosa by immunofluorescence. The relationship between them was determined, and the results can serve as a foundation for future explorations of dysosmia in rhinosinusitis.

\section{MATERIAL AND METHODS}

\section{Experimental animals}

One hundred 6-week-old healthy male Sprague-Dawley rats weighing 180-200 g were selected for the experiments (Experimental Animal Center, Medical College of Nanchang University, Nanchang, China). The rats were divided into the control $(\mathrm{N}=20)$ and experimental $(\mathrm{N}=80)$ groups with a random-number table. None of the animals had any nasal or upper respiratory diseases. This study was conducted in strict accordance with the recommendations in the Guide for the Care and Use of Laboratory Animals of the National Institutes of Health. The animal use protocol was reviewed and approved by the Institutional Animal Care and Use Committee of the First Affiliated Hospital of Nanchang University (Nanchang, China).

\section{Animal models}

The animal model was established with Merocel (Medtronic, Inc., Minneapolis, MN, USA) according to the literature (Ye et al., 2006). In the experimental group, $2 \mathrm{~mm} \times 3 \mathrm{~mm}$ x 20 $\mathrm{mm}$ Merocel sticks were put into the left nasal cavity, and $0.1 \mathrm{~mL}$ Streptococcus pneumoniae was dripped in the Merocel. S. pneumoniae (provided by the Department of Laboratory Medicine, First Affiliated Hospital of Nanchang University) was diluted to $3 \mathrm{McFarland}$ turbidities with sterile saline. The rats were then sacrificed after 1, 2, 3, or 4 weeks (20 rats per group). In the control group, the rats were dripped with saline only; the rats were killed in the 1st week. After an intraperitoneal injection of $3 \mathrm{~mL} / \mathrm{kg}$ chloral hydrate $(10 \%)$ and cervical dislocation, the mandible 
was cut and fixed with 4\% paraformaldehyde for $24 \mathrm{~h}$. Under a dissecting microscope, the nasal cavity and sinus mucosa containing the olfactory mucosa were stripped. Some samples were subjected to dehydration, embedding, staining, and paraffin sectioning. The remaining tissues were prepared as $5-\mu \mathrm{m}$-thick frozen sections, with each group sequentially numbered from 1 to 10 .

\section{Olfactory function tests}

The buried food pellet test (BFPT) proposed by Nathan et al. (2004) was used to detect the olfactory function of the rats. The BFPT of the experimental rats was examined after modeling for 1, 2, 3, and 4 weeks. At each time point, 20 rats were randomly examined after the Merocel sticks were removed from the nasal cavity. The BFPT of the control rats was examined in the first week. The rat diet was restricted by giving them $0.5 \mathrm{~g}$ food every $24 \mathrm{~h}$, and free drinking water. In the BFPT, a container was covered with 3 -cm-thick dressing. Food was buried $0.5 \mathrm{~cm}$ below the surface at random sites. The rats were placed in the test container, and the duration from caging to finding the food (forelimbs clinging to the food pellet or gripping it with teeth) was recorded. Rats that took longer than $15 \mathrm{~min}$ to find the food were removed. Each rat was tested daily in the morning and afternoon at a 6-h interval, and the average duration was used for analysis. The reference standard of rat olfactory dysfunction was a $>300$-s duration, as proposed by Lin et al. (2008). The SPSS13.0 statistical program (IBM Corporation, Armonk, NY, USA) was used for the homogeneity of variance tests. If unequal variances existed, the Dunnett T3 test was used.

\section{Morphological observations}

For the control group and the 1-, 2-, 3-, and 4-week postinoculation groups, $10 \mathrm{sec}-$ tions from each group were used for hematoxylin and eosin (HE) staining.

\section{Olfactory marker protein (OMP) immunofluorescence labeling}

A rabbit anti-rat OMP antibody (SC-67219) was purchased from Santa Cruz Biotechnology, Inc. (Santa Cruz, CA, USA). The 1st and 10th frozen sections, cut by a freezing microtome (Leica Biosystems Nussloch GmbH, Nussloch, Germany), were selected. Thereafter, the sections were washed with phosphate-buffered saline (PBS) and then fixed by $4 \%$ paraformaldehyde for 15 min. Blocking was performed with $0.1 \%$ Triton X-100 (OriGene Technologies, Inc., Rockville, MD, USA) at room temperature for $15 \mathrm{~min}$. After adding goat serum (OriGene Technologies, Inc.), the sections were incubated at $37^{\circ} \mathrm{C}$ for $30 \mathrm{~min}$. Anti-OMP polyclonal antibody (1:400) was added to the sections at room temperature; all sections were kept at $4^{\circ} \mathrm{C}$ overnight. The sections were washed again with PBS, a goat anti-rabbit TRITC secondary antibody (1:200; OriGene Technologies, Inc.) was added at room temperature, and the mixture was incubated in dark for $30 \mathrm{~min}$. Glycerol (1:1) was used for mounting, and an inverted fluorescence microscope (Olympus Corporation, Tokyo, Japan) was used to analyze the results.

\section{p75NTR and glial fibrillary acidic protein (GFAP) double immunofluorescence}

The 2nd to 9th frozen sections were selected. The sections were first washed with PBS 
and then fixed by $4 \%$ paraformaldehyde. Blocking was performed with $0.1 \%$ Triton X-100 at room temperature for $15 \mathrm{~min}$. After adding goat serum, the sections were incubated at $37^{\circ} \mathrm{C}$ for $30 \mathrm{~min}$. Mouse anti-rat p75NTR (1:300; Santa Cruz Biotechnology, Inc.) was added to the sections at room temperature. All sections were kept at $4^{\circ} \mathrm{C}$ overnight and then washed with PBS. A goat anti-mouse FITC antibody (1:400; OriGene Technologies, Inc.) was added at room temperature, and the sections were incubated in dark for $30 \mathrm{~min}$. PBS washes were repeated three times in dark. Triton X-100 (0.1\%) was added for blocking at room temperature for $15 \mathrm{~min}$ in dark. Rabbit anti-rat GFAP (OriGene Technologies, Inc.) (1:200) was added to the sections at room temperature. All sections were kept at $4{ }^{\circ} \mathrm{C}$ overnight. Goat anti-rabbit TRITC antibody (1:100) was added at room temperature, and the sections were incubated in dark for 30 min. Glycerol (1:1) was used for mounting, and an Olympus inverted fluorescence microscope was used to analyze the results.

\section{Statistical analysis}

The data are reported as means \pm standard deviation (SD). Statistical analysis was performed with SPSS 17.0. One-way analysis of variance (ANOVA) was conducted for the comparisons among the different groups. The variances showed heterogeneity and, thus, Dunnett T3 test was used to analyze the differences among the five groups. $\mathrm{P}<0.05$ and $\mathrm{P}<0.01$ were considered to be statistically significant and highly statistically significant, respectively.

\section{RESULTS}

\section{Effect of acute rhinosinusitis on rat olfaction and the olfactory mucosa}

After inoculation of the bacteria in the first few days, some of the experimental rats (36 rats) had scratched noses and sneezed, and some rats (12 rats) had lost appetites and reduced activities. They returned to normal on the fourth day at the latest. The remaining rats had no apparent discomfort. In the control group, nose scratching was observed after drop-bydrop addition of saline, and this symptom disappeared after $1 \mathrm{~h}$ at the latest deaths occurred.

\section{Behavioral test results of olfactory function}

The BFPT results are shown in Figure 1. After an examination of the ANOVA homogeneity, given that an unequal-variance Dunnett T3 test was performed, the durations of finding food were longer in all of the experimental groups compared to the control group $(\mathrm{P}<$ 0.01 ). The longest duration was observed in the 2-week post-inoculation group, and all of the durations in the first 3 weeks exceeded $300 \mathrm{~s}$. The shortest ( $<300 \mathrm{~s})$ was in the 4 -week postinoculation group.

\section{HE staining results}

An Olympus optical microscope was used to examine the HE staining changes in the mucosa, and the results are shown in Figure 2. In the control group, there were regular epithelial cells, complete cilia, no inflammatory cell infiltration, no erythrocytes, no necrotic tissues, 
and no dilated vessels, and the epithelial thickness was normal. In the 1-week post-inoculation group, there were visible gland hyperplasia, vasodilation, tissue edema, erythrocyte infiltrations, and more neutrophil infiltrations in the nasal mucosa, and the epithelium was thin. In the 2-week post-inoculation group, the nasal mucosal gland hyperplasia and tissue edema were reduced slightly, and there were neutrophil infiltrations. In addition, the epithelial layer was thinner, there was almost no cilia growth, and there were exudative necrosis substances in the sinus. In the 3-week post-inoculation group, an obvious mitigated inflammatory response and epithelial proliferation with a disordered arrangement were visible. In the 4-week postinoculation group, the inflammation had almost disappeared, and the epithelium was generally restored. However, there were still a few inflammatory cells in the nasal mucosa.

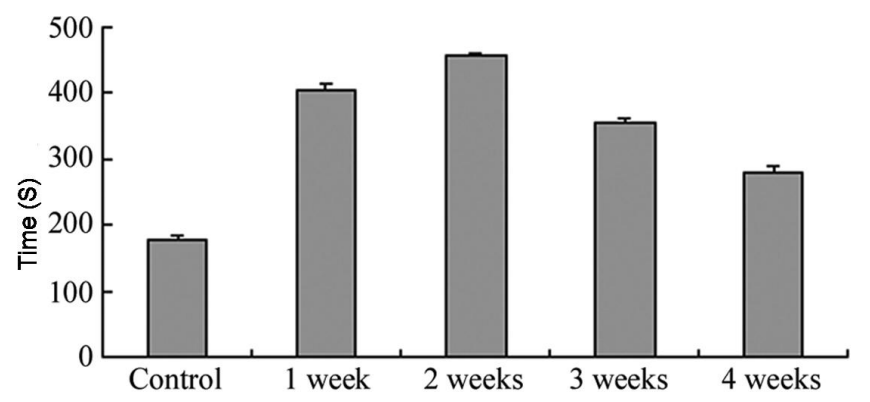

Figure 1. Comparisons among the durations of foraging time of the rats in each group ( $\mathrm{N}=20$ per group).

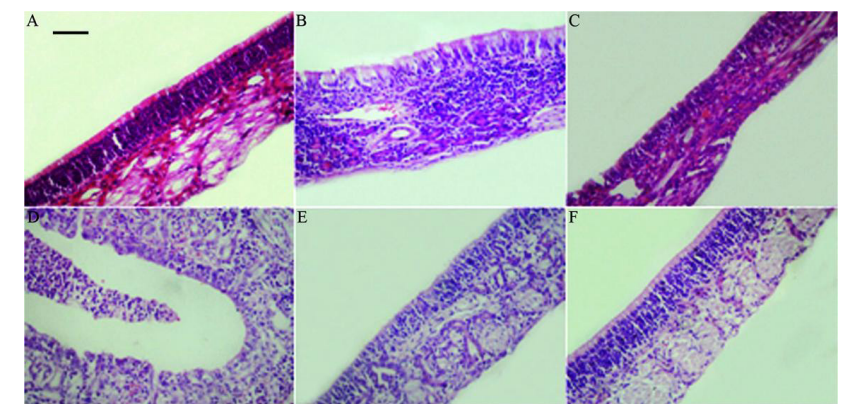

Figure 2. Histopathological examination of the rat sinuses using hematoxylin and eosin staining. A. Control. B. One week post-inoculation. C. Two weeks post-inoculation. D. Two weeks post-inoculation. E. Three weeks postinoculation. F. four weeks post-inoculation (scale bar $=40 \mu \mathrm{m}$ ).

\section{OMP staining and p75NTR-GFAP double-labeling results}

The OMP staining results are shown in Figure 3. The P75NTR-GFAP double-labeling results are shown in Figure 4. S. pneumoniae infection in the rat nasal cavity led to olfactory mucosal and epithelial layer damage with significantly decreased OECs. At the same time, the number of mature ORNs decreased and even disappeared. After the inflammation subsided, the olfactory mucosa was gradually restored, and the recovery of the number of OECs was faster than that of mature ORNs. 

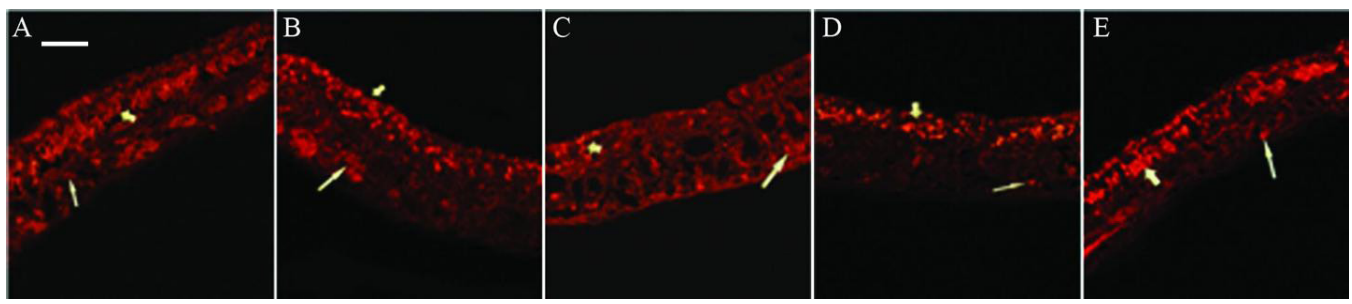

Figure 3. Olfactory marker protein (OMP) staining of the olfactory mucosa. A. Control. There were a large number of red fluorescent regular round or oval mature olfactory receptor neurons (ORN) in the middle and lower olfactory epithelium. B. One week post-inoculation. The olfactory mucosa was disordered, the epithelium was thinning, and the number of mature ORNs was reduced significantly. C. Two weeks post-inoculation. The olfactory mucosa is damaged severely, with epithelial defects appearing, and there were few mature ORNs. D. Three weeks post-inoculation. The thickness of the olfactory mucosa was increased, and more mature ORNs were seen, but with a disordered arrangement. E. Four weeks post-inoculation. The olfactory epithelial thickness had almost returned to normal, and in the middle and lower epithelium, there were more mature ORNs (scale bar $=40 \mu \mathrm{m}$ ). The long arrow points the ORN in the middle and lower olfactory epithelium, the short arrow point the ORN in the upper olfactory epithelium.
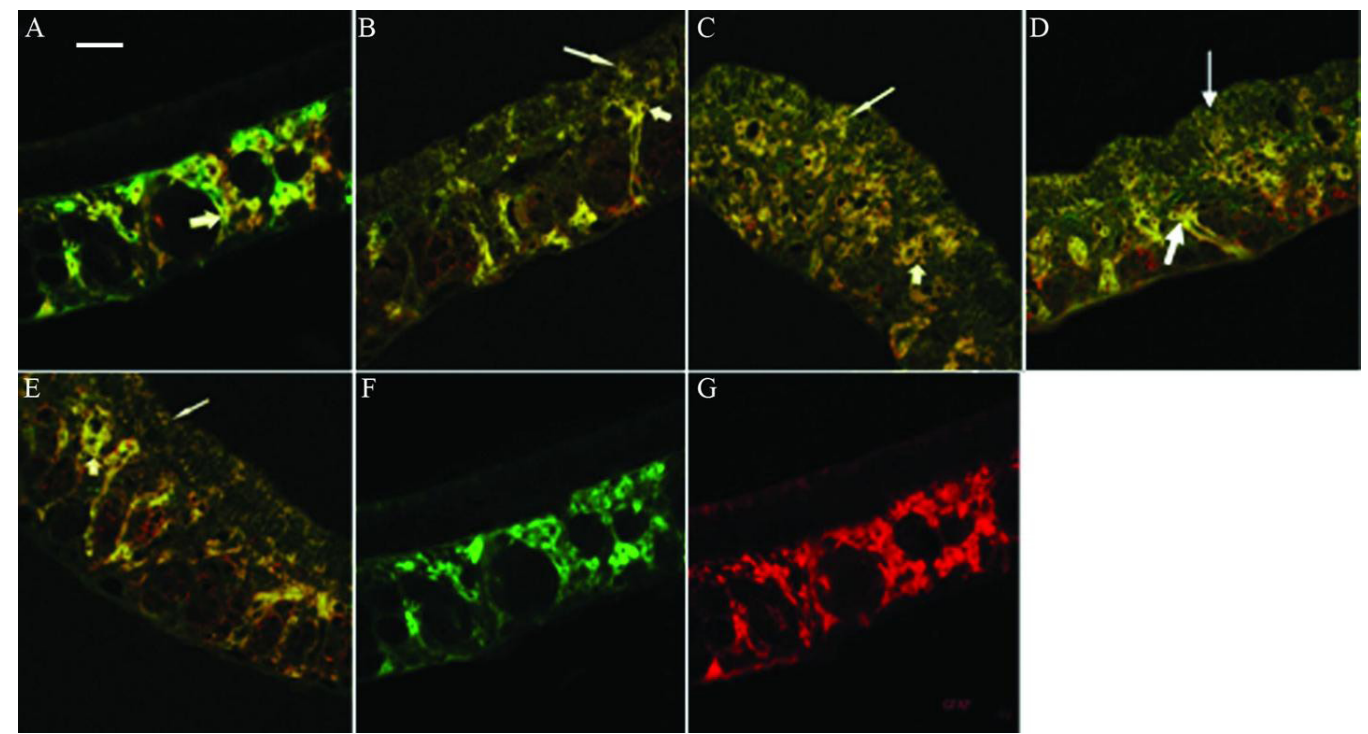

Figure 4. p75NTR and glial fibrillary acidic protein (GFAP) double-labeling immunofluorescence staining. A. Control. The lamina propria of the olfactory mucosa, close to the basement membrane, exhibited a large number of yellow fluorescence-lined fusiform or oblate olfactory ensheathing cells (OEC) (thick arrow), and spindle cells were the majority. B. One week post-inoculation. The olfactory epithelium was thinning, and the OECs were reduced significantly (thick arrow) in the lamina propria. In the olfactory epithelium (thin arrow), OECs grew occasionally. C. Two weeks post-inoculation. The olfactory epithelium was significantly thinner. OECs were increased more than the previous week (thick arrow) in the lamina propria. In the olfactory epithelium, there was a small amount of OEC (thin arrow). D. Three weeks post-inoculation. The thickness of the olfactory epithelium was increased. A part of the increased OEC formed a colony (thick arrow) in the lamina propria. There was a small amount of OECs (thin arrow) in the olfactory epithelium. E. Four weeks post-inoculation. The olfactory epithelium was generally recovered to normal. OECs were almost restored (thick arrow) in the lamina propria. In the olfactory epithelium, OECs grew occasionally (thin arrow). F. p75NTR immunofluorescence labeling protocol. G. GFAP immunofluorescence labeling protocol (scale bar $=40 \mu \mathrm{m}$ ). 


\section{DISCUSSION}

Rhinosinusitis is one of the main reasons for dysosmia. After medical treatment and/ or surgery, a considerable part of the patient's olfaction may fail to improve. These phenomena are theoretically difficult to explain by ORN regeneration and OEC-promoted ORN regeneration. The local inflammation of nasal sinuses, especially the olfactory cleft, hinders ORN regeneration and may affect the growth and survival of OECs. Most studies have focused on the effects of rhinosinusitis on ORNs, and reports on OEC changes in rhinosinusitis are uncommon (Yee et al., 2010). In this study, we established a rat acute rhinosinusitis model and detected mature ORN and OEC changes in the olfactory mucosa using immunofluorescence. The acute inflammation led to a significant decrease in ORNs and OECs, and the recovery of the OECs was earlier than that of the ORNs. This phenomenon suggested that acute inflammation significantly affected the ORNs and OECs. However, in the case of co-instantaneous damage, the recovery of the OECs was more rapid than that of the ORNs. These results provide the basis for our next study on the impact of chronic inflammation on ORNs and OECs.

The obstruction of the ostiomeatal complex and bacterial infection are key factors of rhinosinusitis (Brook, 2007). The virulence of S. pneumoniae increases in pathological states; this enables the bacteria to invade the mucosa, thereby causing mucosal injury and disease. The HE staining results showed that, despite the discrepant findings of local and international studies on the degree of sinonasal mucosal inflammatory reactions, the overall trends of the inflammatory responses were similar.

The BFPT is the most common olfactory behavior detection method. The principle is that rats are animals with olfactory acuity. Thus, when foods are buried, their foraging mainly relies on the sense of smell. The duration from caging until finding the food can reflect olfactory function. The BFPT was suggested by Nathan et al. (2004) for the estimation of rat olfactory function. The dysfunction reference standard is a duration of $>300 \mathrm{~s}$, as proposed by Lin et al. (2008).

The results showed that the durations were longer in the experimental group than in the control group, and the differences were significant. Three weeks after inoculation, the durations of foraging were more than $300 \mathrm{~s}$, demonstrating that the rats had various degrees of olfactory dysfunction. Four weeks post-inoculation, the duration was $<300 \mathrm{~s}$, but it was still longer than that of the control group. Combined with the HE staining results, the olfactory damage can be inferred to be related to the degree of inflammatory reaction, which means that a heavier inflammatory reaction results in longer foraging duration and a more serious degree of dysosmia. The above results indicated that a rhinosinusitis-caused dysosmia rat model was successfully established.

ORNs are located in the middle and bottom parts of the olfactory epithelium. Under physiological conditions, a large number of neuron-pathogenic microorganisms exist in the nasal cavity, and when the body resistance decreases or during dysbacteriosis, neuronal damage and apoptosis can be induced by inflammatory responses (Jia et al., 2011). In our study, S. pneumoniae, which is the common cause of rhinosinusitis, was selected for establishing a model that is close to the development of human rhinosinusitis. OMP is only expressed in mature ORNs and in the olfactory nerve bundle of the lamina propria (Lee et al., 2011). In the present study, the changes in the mature ORNs were recorded by immunofluorescence.

The results showed that mature ORN expression decreased in all of the experimental groups. The reduction started from the 1st week and peaked in the 2 nd week. Afterwards, 
mature ORNs began to increase, and the thickness of the olfactory epithelium also increased. In the fourth week, mature ORNs generally returned to normal levels. Combined with reports in the literature, rat nasal packing combined with the inoculation of $S$. pneumoniae can induce acute rhinosinusitis. In the olfactory epithelium, the reduction in mature ORNs was related to the pathological severity and the degree of dysosmia ( $\mathrm{Su}$ and He, 2010). However, with the regression of the inflammation, the mature ORNs and mucosal regeneration increased, and the regenerated olfactory mucosa retained olfactory function.

In patients with dysosmia caused by rhinosinusitis, mature ORNs are significantly decreased (Yee et al., 2009). A study has also confirmed that ORN regeneration requires the proliferation of precursor cells (Iwai et al., 2008) and the support of various neurotrophic factors (Malnic and Armelin-Correa, 2010). OECs are unique glial cells in the olfactory system, and they have characteristics of both Schwann cells and astrocytes (Su and He, 2010). OECs are the only cells able to express p75NTR in an olfactory system. The GFAP antibody specifically marks glial cells, and OECs are sensitive to GFAP and show uniform staining in the embryonic stage, neonatal phase, and adulthood (Liu et al., 2010). Our results showed that, 1 week after inoculation, the OECs were significantly decreased on the vaccination side, but, after the 2nd week, the OECs increased earlier than the ORNs. However, a polarity disorder was observed. In the $3 \mathrm{rd}$ week, numerous OECs were found, and the polarity was restored. In the fourth week, the OECs generally returned to normal and were arranged in neat rows. According to a previous study ( $\mathrm{Su}$ and $\mathrm{He}, 2010)$, the apoptosis of mature ORNs increases acute rhinosinusitis and reduces OECs transiently. However, along with the initial increase in OECs, the inflammatory response peak became most obvious in the 2nd week. After the degradation of inflammation, the normal levels of OECs were gradually restored.

During rhinosinusitis, the numbers of ORNs and OECs were not synchronous for the following reasons. First, a severe inflammation speeds up mature ORN apoptosis, inhibits the maturity of neurons, and increases the immune cell irritability of OECs earlier than ORNs, all of which prevent immune surveillance (Harris et al., 2009). A severe inflammation can also be directly involved in the phagocytosis and internalization of pathogens (Leung et al., 2008) and promote an earlier recession of inflammation. Second, OECs play a role that is similar to Schwann cells during inflammation, and proliferation increases to form a Bungner band and limit the inflammation. Third, inflammation damages the olfactory nerve, reduces nerve growth factors and other substances in OECs and the olfactory bulb, and causes dysmaturity of newborn ORNs. An earlier recovery of OECs is conducive to the release of neurotrophic factors for the regeneration of damaged axons (Malnic and Armelin-Correa, 2010). OECs can also swallow apoptotic ORNs and participate in the removal of axon debris (Li et al., 2005).

We also found that OECs grew in the olfactory epithelium during inflammation. This finding differed from those of local and international reports (Williams et al., 2004). The possible reasons for this were as follows. First, S. pneumoniae can identify the OEC surface mannose receptor and is located in OECs (Macedo-Ramos et al., 2011). OECs can also play an immune surveillance role and migrate (Williams et al., 2004) through the basement membrane (Windus et al., 2007). Phagocytosis, internalization, and eradication of S. pneumoniae occur and, thus, OECs play a similar role as phagocytes (Leung et al., 2008). Second, a study has confirmed that OECs originate from original ORNs in olfactory mucosa and are accompanied by original ORN growth and migration (Ramon-Cueto and Avila, 1998). OECs may directly mature in the olfactory epithelium, protect ORNs, and clear S. pneumoniae. The underlying mechanism requires further research. 
Dysosmia is the most common complaint of rhinosinusitis, but only some patients improve after treatment (Silas and Doty, 2010). ORNs are the basic unit of an olfactory system, whereas OECs play an important role in ORN regeneration and maturity. The underlying mechanisms can help us gain further insight into dysosmia that is caused by rhinosinusitis and establish possible olfactory function treatment and recovery strategies.

\title{
ACKNOWLEDGMENTS
}

\author{
Study supported by the Natural Science Foundation of Jiangxi Province \\ (\#2008gqy0034).
}

\section{REFERENCES}

Brook I (2007). Acute and chronic bacterial sinusitis. Infect. Dis. Clin. North Am. 21: 427-48, vii.

Harris JA, West AK and Chuah MI (2009). Olfactory ensheathing cells: nitric oxide production and innate immunity. Glia 57: 1848-1857.

Iwai N, Zhou Z, Roop DR and Behringer RR (2008). Horizontal basal cells are multipotent progenitors in normal and injured adult olfactory epithelium. Stem. Cells 26: 1298-1306.

Jia C, Sangsiri S, Belock B, Iqbal TR, et al. (2011). ATP mediates neuroprotective and neuroproliferative effects in mouse olfactory epithelium following exposure to satratoxin G in vitro and in vivo. Toxicol. Sci. 124: 169-178.

Lee AC, He J and Ma M (2011). Olfactory marker protein is critical for functional maturation of olfactory sensory neurons and development of mother preference. J. Neurosci. 31: 2974-2982.

Leung JY, Chapman JA, Harris JA, Hale D, et al. (2008). Olfactory ensheathing cells are attracted to, and can endocytose, bacteria. Cell Mol. Life Sci. 65: 2732-2739.

Li Y, Field PM and Raisman G (2005). Olfactory ensheathing cells and olfactory nerve fibroblasts maintain continuous open channels for regrowth of olfactory nerve fibres. Glia 52: 245-251.

Lin J, Wei YX and Wang XD (2008). Observation of the olfactory mucosa in mice with allergic rhinitis olfactory dysfunction. China Arch. Otolaryngol. Head Neck Surg. 15: 465-468.

Liu K, Li Y, Wang H, Jiang X, et al. (2010). The immunohistochemical characterization of human fetal olfactory bulb and olfactory ensheathing cells in culture as a source for clinical CNS restoration. Anat. Rec. 293: 359-369.

Macedo-Ramos H, Campos FS, Carvalho LA, Ramos IB, et al. (2011). Olfactory ensheathing cells as putative host cells for Streptococcus pneumoniae: evidence of bacterial invasion via mannose receptor-mediated endocytosis. Neurosci. Res. 69: 308-313.

Malnic B and Armelin-Correa L (2010). Neurogenesis in the Olfactory Epithelium. In: Perspectives of Stem Cells. (Ulrich H, ed.). Springer, Berlin, 35-45.

Nathan BP, Yost J, Litherland MT, Struble RG, et al. (2004). Olfactory function in apoE knockout mice. Behav. Brain Res. 150: 1-7.

Raisman G and Li Y (2007). Repair of neural pathways by olfactory ensheathing cells. Nat. Rev. Neurosci. 8: 312-319.

Ramon-Cueto A and Avila J (1998). Olfactory ensheathing glia: properties and function. Brain Res. Bull. 46: 175-187.

Shetty RS, Bose SC, Nickell MD, McIntyre JC, et al. (2005). Transcriptional changes during neuronal death and replacement in the olfactory epithelium. Mol. Cell Neurosci. 30: 583-600.

Shipley MT, Ennis M and Puche AC (2008). The Olfactory System. In: Neuroscience in Medicine. (Conn PM, ed.). Humana Press, Totowa, 611-622.

Silas J and Doty RL (2010). No evidence for specific benefit of acupuncture over vitamin B complex in treating persons with olfactory dysfunction. Otolaryngol. Head Neck Surg. 143: 603-604.

$\mathrm{Su} \mathrm{Z}$ and $\mathrm{He} \mathrm{C}$ (2010). Olfactory ensheathing cells: biology in neural development and regeneration. Prog. Neurobiol. 92: 517-532.

Williams SK, Gilbey T and Barnett SC (2004). Immunohistochemical studies of the cellular changes in the peripheral olfactory system after zinc sulfate nasal irrigation. Neurochem. Res. 29: 891-901.

Windus LC, Claxton C, Allen CL, Key B, et al. (2007). Motile membrane protrusions regulate cell-cell adhesion and migration of olfactory ensheathing glia. Glia 55: 1708-1719.

Ye J, Yu HM and Li HW (2006). Combination of merocel sponge with streptococcus pneumoniae to establish rat model of acute bacterial rhinosinusitis. China J. Ophthalmol. Otorhinolaryngol. 6: 214-216. 
Yee KK, Pribitkin EA, Cowart BJ, Rosen D, et al. (2009). Analysis of the olfactory mucosa in chronic rhinosinusitis. Ann. N. Y. Acad. Sci. 1170: 590-595.

Yee KK, Pribitkin EA, Cowart BJ, Vainius AA, et al. (2010). Neuropathology of the olfactory mucosa in chronic rhinosinusitis. Am. J. Rhinol. Allergy 24: 110-120. 